\title{
Eikonal wave functions and model independent exclusive $B$ decays
}

\author{
R. Akhoury ${ }^{a, 1}$, I.Z. Rothstein ${ }^{b}$ \\ a Physics Department, Brookhaven National Labs, Long Island, NY 11973, USA \\ b The Randall Laboratory of Physics, University of Michigan, Ann Arbor, MI 48109, USA \\ Received 18 July 1994 \\ Editor: H. Georgi
}

\begin{abstract}
In this letter we calculate the proper normalization for the $B$ meson eikonal valence wave function used in exclusive $B$ decays. The normalization appropriate for a hard scattering process renormalized at the scale $\mu$ is determined by calculating the short distance contribution to the purely leptonic decay.
\end{abstract}

\section{Introduction}

Recently, it has been proposed that exclusive $B$ meson decay amplitudes may be calculated in a consistent fashion. In [1] it was shown that the form factor for the the $B$ meson decay into a pion may be written in the form

$$
\begin{aligned}
& M\left(p, p^{\prime}\right)=\int \frac{d^{4} k}{(2 \pi)^{4}} \\
& \quad \times \int_{0}^{1} d \xi \phi_{B}(k) H\left(k, \xi, p, p^{\prime}\right) \phi_{\pi}(\xi),
\end{aligned}
$$

where $\phi_{B}(k)$ and $\phi_{\pi}(\xi)$ are the $B$ and $\pi$ meson valence wavefunction respectively, and $H$ is the hard scattering amplitude. To make the calculation of the hard scattering amplitude consistent it is necessary to factorize the infrared divergences coming from soft and collinear gluon. Once factorization has been proven the infrared divergences may be absorbed into

\footnotetext{
1 On leave of absence from the Univ. of Michigan, Ann Arbor.
}

the the wavefunction, which contains all the long distance physics.

In [1] the divergences from soft gluon exchange were shown to sum into an eikonal phase which may be absorbed into the $B$ meson wavefunction. Physically, this summation is a consequence of the the fact that in the low energy effective theory the heavy quark acts as a static color source. Furthermore, the collinear divergences were shown to be cutoff due to higher order Sudakov corrections [2]. Once it is known that the infrared divergences are factorizable (in the case of the soft divergences) or cutoff (in the case of the mass singularities), the next step in the justification of the use of (1), is to show that the two particle wave function dominates the decay amplitude. In the case of $B$ decays, the contribution from states with larger number of partons will be down by $O\left(\Lambda / m_{b}\right)$.

In [1] the dependence of the wave-function on the heavy quark mass was left undetermined. In this letter we calculate the scale dependence of the universal eikonal $B$ meson wavefunction. This study is motivated by the fact that wave function must be appropriately normalized. In general, the normalization will 


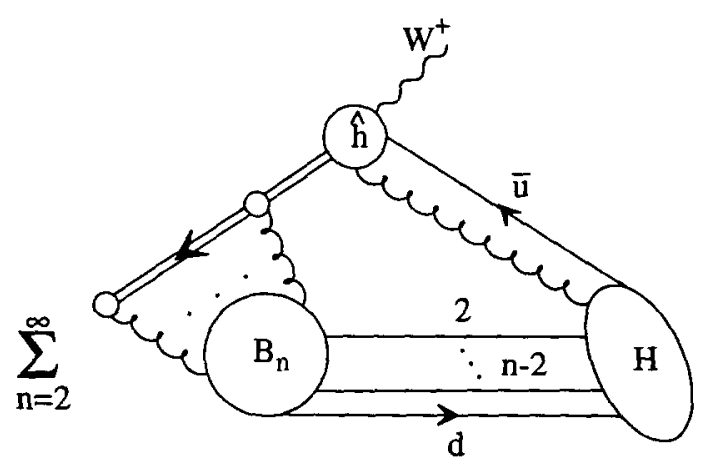

Fig. 1. Spacetime picture of the semi-leptonic decay of a $B$ meson into some final hadronic state $\mathrm{H}$.

depend upon the hard scattering amplitude which is of course process dependent. To lowest order in $\alpha_{s}$ and $\Lambda / m_{b}$ the normalization is given by ${ }^{2}$

$$
\int d k^{-} \hat{\phi}_{B}\left(k^{-}\right)=\frac{1}{2 \sqrt{3}} f_{B},
$$

and we have defined

$$
\hat{\phi}_{B}\left(k^{-}\right)=\int d k^{+} d^{2} k_{\perp} \phi_{B}(k) \text {. }
$$

This normalization, defined through the purely leptonic decay, includes contribution from short (compared to the QCD scale) wavelength gluons which may also contribute to the hard scattering amplitude. Thus, to avoid double counting the contribution from these hard gluons, we would like to determine a scale dependent normalization for the wave function. In other words, it is necessary to extract the short distance contributions to the normalization of the wave function from the purely leptonic decay.

\section{The eikonal wave function}

Fig. 1 illustrates the generic structure for a $B$ meson decay. The $B$ meson is composed of $n$ incoming partons, one of which, the $b$ quark, decays into a light quark. $h$ labels the hard scattering amplitude, and the final blob represents the hadronization of the final state partons into the final state of choice. For our purposes we will assume that the two parton state dominates the decay process. In general, this must be

\footnotetext{
2 This normalization corresponds to $f_{\pi}=93 \mathrm{MeV}$.
}

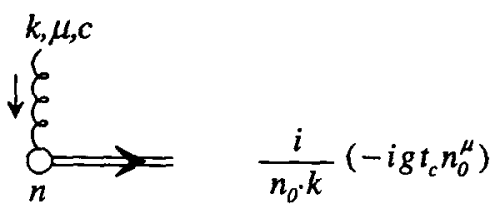

Fig. 2. Feynman rules for eikonal vertices.

shown to be due to some dynamics. As discussed in the introduction, to make the calculation of the decay amplitude consistent it is necessary to show that the divergences due to soft gluon exchange can be factorized and placed into the wave function. The eikonal wave function is defined in a manner so as to absorb all such soft divergences, as we will now show.

Consider the absorption/emission of a soft gluon from the heavy quark line. Heavy quark effective field theory [3] (HQET) tells us that the heavy quark will be off shell by at most $O\left(\Lambda_{\mathrm{QCD}}\right)$. Thus, for gluon with momenta satisfying the constraint $q^{2}<\Lambda_{\mathrm{QCD}} m_{b}$, we may use the Feynman rules expressed in Fig. 2. As in HQET, the heavy quark propagator satisfies the constraint

$\frac{1}{2}(1+\not b) h_{v}=h_{v}$.

Using these Feynman rules we may factor out all soft gluon exchanges from the hard scattering amplitude [1]. This can be seen by going to the rest frame of the heavy quark and resumming all the soft gluons into a path ordered non-abelian eikonal phase defined by

$U\left(A^{0}\right)=P \exp \left(-i g \int_{-\infty}^{0} A^{0}\left(\lambda n_{0}\right) d \lambda\right)$.

In this definition $n_{\mu}$ is the unit four-vector in the direction of the heavy quark four velocity.

By inserting $U U^{-1}$ into the matrix element relevant for the decay under study, we may systematically remove the divergences due to soft gluon exchanges to all orders by absorbing the eikonal phase into the wave function. In this way we may write the gauge invariant two particle wave function as

$\psi_{B}(x)=\left\langle 0\left|T\left(\bar{b}^{\text {free }}(0) U\left(A^{0}\right) q(x)\right)\right| B(p)\right\rangle_{L(q)}$,

where the interaction lagrangian $L(q)$, is independent of the $b$ quark field. In the above a trace over color in- 


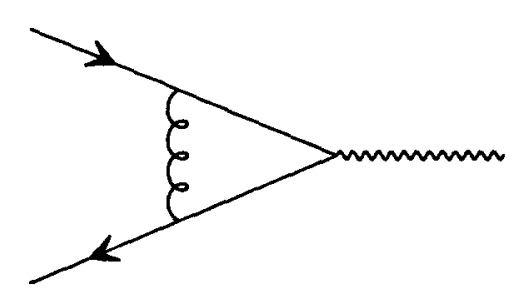

(a)

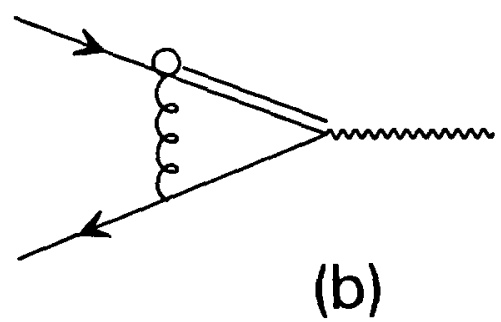

Fig. 3. (a) The full annihilation vertex. (b) The eikonal annihilation vertex.

dices is assumed. Furthermore, the gauge invariance of the wave function is made manifest via the introduction of an additional path ordered Schwinger phase. The contribution to the decay process from this additional phase factor is higher twist and will thus be neglected. This definition incorporates the decoupling of the heavy quark, in that there are no $b$ quark loops contributing to the amplitude. By using this wave function the hard scattering amplitude becomes manifestly free of soft divergences as a consequence of the fact that for every diagram with a gluon exchange we must subtract the same diagram only using the heavy quark Feynman rules defined in Fig. 2.

\section{Normalization of the eikonal wave function}

To make contact between physical quantities and the above mentioned wave function, it is necessary to normalize the wave function to $f_{B}$, up to some constant through the purely leptonic decay. The lowest order diagram is shown in Fig. 3, giving the normalization in Eq. (2). However, to make this normalization process independent we should calculate the next order correction so that we may remove the contribution from gluons with momenta large compared to the QCD scale. This entails calculating the corrections to the difference between the full and eikonalized currents. Here we present the leading order in $\Lambda_{\mathrm{QCD}} / m_{b}$ calculations for both the full and eikonalized renormalized currents, as shown in Figs. 3a, 3b. In the results presented below we always project onto the pseudoscalar color singlet state, and we choose to work in the Feynman gauge.

The hadronic part of the amplitude is given by

$$
\left\langle 0\left|J_{\mu}^{h}\right| B(p)\right\rangle=\sqrt{2} i f_{B} p_{\mu},
$$

where we have defined

$J_{\mu}^{h}=-i \bar{b} \gamma_{\mu}\left(1-\gamma_{5}\right) u$.

This amplitude may also be written as

$\int \frac{d^{4} k}{(2 \pi)^{4}} \phi_{B}(k, \mu) H_{\mu}(p, k, \mu)$.

The hard scattering amplitude $H_{\mu}(p, k, \mu)$ is projected onto the pseudoscalar color singlet channel. To lowest order it is given by (see Fig. 1)

$H_{0}(p, k)=\frac{N_{c}}{\sqrt{6}} 4 i_{b}$,

in the rest frame of the meson. In this equation, as well as in the rest of the paper, we do not distinguish between the mesonic and heavy quark mass.

In this frame the one loop renormalized full vertex is given by

$$
\begin{aligned}
& \frac{1}{\sqrt{6}} Z_{l}^{1 / 2} Z_{h}^{1 / 2} \operatorname{Tr}\left(\gamma_{5}\left(\not p+m_{b}\right)\left\langle 0\left|J_{\mu}\right| b \bar{u}\right\rangle\right) \\
& \quad=\frac{N_{c}}{\sqrt{6}} \frac{i \alpha_{s} m_{b}}{3 \pi}\left[-2 \ln \frac{-k^{2}}{m_{b}^{2}}-8 \ln \frac{2 p \cdot k}{m_{b}^{2}}+4\right] .
\end{aligned}
$$

The wave function renormalizations are given by

$$
\begin{aligned}
Z_{l}^{1 / 2} & =1-\frac{\alpha_{s}}{6 \pi}\left[\frac{1}{\epsilon}-\gamma-\ln \frac{-k^{2}}{4 \pi \mu^{2}}-1\right] \\
Z_{h}^{1 / 2} & =1 \\
- & \frac{\alpha_{s}}{6 \pi}\left[\frac{1}{\epsilon}-\gamma-\ln \frac{m_{b}^{2}}{4 \pi \mu^{2}}+4 \ln \frac{2 p \cdot k}{m_{b}^{2}}+4\right] .
\end{aligned}
$$

The one loop renormalized eikonal vertex is 


$$
\begin{aligned}
& \frac{1}{\sqrt{6}} Z_{l}^{1 / 2} Z_{Q}^{1 / 2} \operatorname{Tr}\left(\gamma_{5}\left(\not p+m_{b}\right)\left\langle 0\left|J_{\mu}\right| b \bar{u}\right\rangle\right) \\
& \quad=\frac{N_{c}}{\sqrt{6}} \frac{i \alpha_{s} m_{b}}{3 \pi}\left[\frac{6}{\epsilon}-10 \gamma-2 \ln \frac{-k^{2}}{4 \pi \mu^{2}}\right. \\
& \left.-4 \ln \frac{(2 n \cdot k)^{2}}{4 \pi \mu^{2}}+14\right] .
\end{aligned}
$$

The eikonal wave function renormalization is

$$
Z_{Q}^{1 / 2}=1-\frac{\alpha_{s}}{3 \pi}\left[\frac{1}{\epsilon}-2 \gamma+1-\ln \frac{(2 n \cdot k)^{2}}{4 \pi \mu^{2}}\right] .
$$

Notice that neither (11) nor (13) contain any double logs which are usually present due to the region of integration where the gluon is both soft and collinear to the light quark. The absence of these logs is a consequence of the fact that we are projecting onto the color singlet state, which doesn't couple to long wavelength gluons. Also, as seen from (13), the eikonal vertex necessitates an infinite counter-term because the eikonal current is no longer partially conserved.

The hard scattering amplitude $H_{\mu}(p, k, \mu)$ in the $B$ meson rest frame is given by the difference between the full and eikonal vertices

$$
\begin{aligned}
& H_{0}(p, k, \mu) \\
& \quad=i \frac{N_{c}}{\sqrt{6}} 4 m_{b}\left[\frac{\alpha_{s}}{6 \pi}\left(3 \ln \frac{m_{b}^{2}}{4 \pi \mu^{2}}-5+5 \gamma\right)\right] .
\end{aligned}
$$

The collinear divergences cancel to all orders in the hard scattering amplitude even though they may come from hard gluons. This is simply because the light quark is treated on the same footing in both the full as well as eikonal theories. Furthermore, the two theories must match at $\mu=m_{b}$.

Combining the lowest order result with (15) leaves

$$
\begin{aligned}
i & \sqrt{2} m_{b} f_{B}=\int \frac{d^{4} k}{(2 \pi)^{4}} \phi_{B}(k, \mu) \frac{N_{c}}{\sqrt{6}}\left(4 i m_{b}\right) \\
& \times\left[1+\frac{\alpha_{s}}{6 \pi}\left(3 \ln \frac{m_{b}^{2}}{4 \pi \mu^{2}}-5+5 \gamma\right)\right] .
\end{aligned}
$$

Thus, the leading order correction to the wave function is given by

$$
\begin{aligned}
f_{B} & =\frac{1}{2 \sqrt{3}} \psi_{B}(0, \mu) \\
& \times\left[1+\frac{\alpha_{s}}{6 \pi}\left(3 \ln \frac{m_{b}^{2}}{4 \pi \mu^{2}}-5+5 \gamma\right)\right],
\end{aligned}
$$

and explicitly shows the separation of the hard gluon contribution to the decay process.

We may resum the leading logs using the renormalization group leaving

$f_{B}=\frac{\psi_{B}(0, \mu)}{2 \sqrt{3}}\left[\frac{\alpha\left(m_{b}\right)}{\alpha(\mu)}\right]^{\frac{-2}{11-2 n^{\prime / 3}}}$.

In this expression the wave function at the origin is independent of the heavy quark mass. Eq. (18) may be used to rederive the result originally found by Voloshin and Shifman [4] for the ratio of the $B$ to $D$ meson decay constants

$$
\frac{f_{B}}{f_{D}}=\frac{\sqrt{m_{c}}}{\sqrt{m_{b}}}\left[\frac{\alpha_{s}\left(m_{b}\right)}{\alpha_{s}\left(m_{c}\right)}\right]^{\frac{-2}{11-2 n_{f} / 3}} .
$$

Where $n_{f}$ is 4 and the dependence on the heavy quark masses comes from normalizing the states in a mass independent fashion.

We would like to point out that the eikonal wave function is a lattice observable [5]. This is an important point given the fact that final state interactions make hadronic decays inaccessible to lattice simulations. Thus, we believe that the method discussed in [1] and above is presently the only model independent method for calculating exclusive decay amplitudes. Furthermore, it should be stressed that like light-cone wave functions, the eikonal wave function will satisfy the usual Bethe-Salpeter type integral equation.

In conclusion it should be mentioned that the formalism, in the form discussed above, does not take into account any non-perturbative effects other than those in the eikonal wave function. These contribution of these effects to the decay of $B$ hadrons into light mesons are presently under study.

The authors benefitted from conversation with A. Falk and G. Sterman. RA would like to thank the Max Planck Institute for Physics, Munich, especially Prof. W. Zimmerman for hospitality and support. IZR gratefully acknowledges the Aspen Center for Physics where part of this work was done.

\section{References}

[1] R. Akhoury, G. Sterman and Y.P. Yao, Exclusive Semileptonic Decays of $B$ Mesons into Light Mesons, UMTH-93-11, ITP-SB-93-25. (To appear in Phys. Rev. D). 
[2] V. Sudakov, JETP (Sov. Phys.) 3 (1956) 65; A.H. Mueller, Phys. Rev. D 20 (1979) 2037; J.C. Collins, Phys. Rev. D 22 (1980) 1478; A. Sen, Phys. Rev. D 24 (1981) 2181; G.P. Korchemskii and A.V. Radyushkin, Sov. J. of Nucl. Phys. 45 (1987) 910.

[3] N. Isgur and M.B. Wise, Phys Lett. B 232 (1989) 113; 237 (1990) 527;
H. Georgi, Phys. Lett. B 240 (1990) 447;

E. Eichten and B. Hill, Phys. Lett. B 234 (1990) 511;

A.F. Falk, H. Georgi, B. Grinstein and M.B. Wise, Nucl. Phys. B 343 (1990) 1.

[4] M.B. Voloshin and M.A. Shifman, Yad. Fiz. 45 (1987); Sov. J. Nucl. Phys. 45 (1987) 292.

[5] C. Bernard, private communication. 